\title{
Performance analysis of Permanent Magnet Assisted reluctance synchronous machine
}

\author{
Fuhui-kai ${ }^{1, a}$ \\ ${ }^{1}$ Mechanical and Electrical Engineering Institute, Xinxiang University, Xinxiang 453003, China \\ afuhuikai@163.com
}

Keywords: Reluctance synchronous machine; flux linkage; Torque response ; Finite Element;

\begin{abstract}
This thesis is about the dynamic control of a permanent magnet assisted reluctance synchronous machine (PMA RSM).The PMA RSM in this thesis is a $110 \mathrm{~kW}$ traction machine and is ideal for the use in electrical rail vehicles. For accurately analyzing the dynamic characteristics paper is of the of a permanent magnet assisted reluctance synchronous machine, For the body of machine, the paper carries on the finite element analysis of its D-Q axis flux linkage, the self inductance, the mutual inductance. The mathematical model of the Permanent Magnet Assisted reluctance synchronous machine is derived and explained in physical terms from analytical data. To improve the dynamic performance of the PMA RSM, a load torque observer with compensation current feedback is investigated. The derivation of the load torque observer and the detail designs of the observer elements are given. The accurate simulation model of the PMA RSM is used to verify the operation of the observer structures and to evaluate the dynamic performance.
\end{abstract}

\section{Introduction}

Permanent magnet synchronous motor has many advantages such as simple structure, small size, light weight and high efficiency, compared to the DC motor, it has no the shortcomings of commutator and brush; And compared to the induction motor, it don't need reactive exciter current, having high efficiency and power factor[1-3]. This novel place of the paper is combination of the characteristics of synchronous reluctance motors and synchronous permanent magnet motor. The new design concept is permanent magnet assisted reluctance synchronous motor. Comparative studies carried out on the performance of the RSM and induction machine in the low- and medium-power range show that the RSM has higher torque density and efficiency[4-5].It was found that the RSM has more advantages than the induction machine, because the absence of rotor currents leads to a simple vector control scheme and insignificant rotor losses. The rotor manufacturing cost for the RSM is relatively low compared to that of the induction machine.

\section{The mathematical model for the PMA RSM}

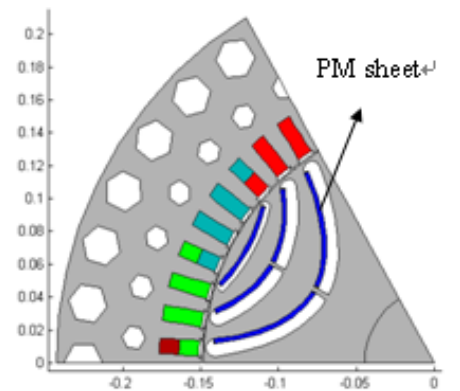

Fig.1 Cross section of the Permanent Magnet Assisted reluctance synchronous machine

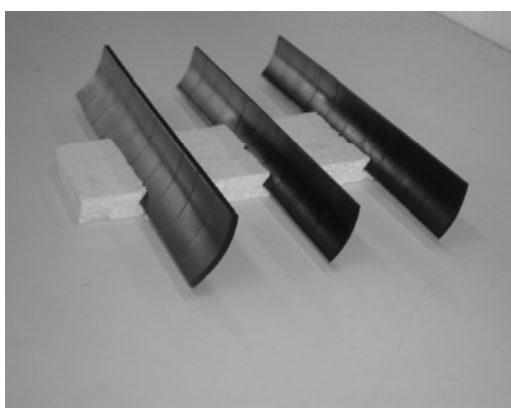

Fig.2 Permanent magnetism board of the Permanent Magnet Assisted reluctance synchronous machine 
It is very necessary to establish the mathematical model of the motor to truly understand its working principle. A cross-section of the RSM with permanent magnet sheets incorporated into the reluctance rotor is shown in Fig.1.the actual PM sheets added in the motor is shown in Fig.2

Since the rotor of the RSM does not contain any windings, the electrical model is based only on the stator. The induced stator voltage is given by Faraday's Law and the copper loss component also has to be taken into account in the voltage equation as follows:

$$
v_{a b c}=\frac{d \lambda_{a b c}}{d t}+r_{s} i_{a b c}
$$

The equation above is in the stationary $\mathrm{ABC}$ reference frame which is fixed to the stator. The stator windings are represented as three stationary coils displaced by $120^{\circ}$ spatially. The model of the machine can be simplified by using Park's transform .The voltage equations in the QD0 reference frame, which is fixed to the rotor, are given by:

The equation above is in the stationary ABC reference frame which is fixed to the stator. The stator windings are represented as three stationary coils displaced by $120^{\circ}$ spatially. The model of the machine can be simplified by using Park's transform. The voltage equations in the QD0 reference frame, which is fixed to the rotor, are given by:

$$
\begin{aligned}
& v_{d}=r_{s} i_{d}+\frac{d \lambda_{d}}{d t}-\omega_{e} \lambda_{q} \\
& v_{q}=r_{s} i_{q}+\frac{d \lambda_{q}}{d t}+\omega_{e} \lambda_{d}
\end{aligned}
$$

The Park transform changes the voltage equations from a stationary ABC reference frame to a synchronously rotating QD0 reference frame. It is a one-to-one mapping that is dependent on the rotor position. Note that the QD0 equations are still as seen from the stator. The stator is now represented by two coils displaced by $90^{\circ}$ spatially. The two stator coils rotate synchronously with the rotor. This representation is shown in Fig.3.

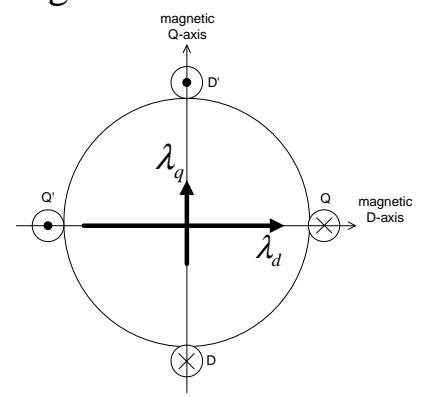

Fig.3 Park transform representation of stator coils

As the rotor position changes caused by the flux are relatively small, The derivation of $\mathrm{d}$ and $\mathrm{q}$ flux linkage are given by:

$$
\begin{aligned}
& \frac{d \lambda_{q}}{d t}=M_{q}^{\prime} \frac{d i_{d}}{d t}+L_{q}^{\prime} \frac{d i_{q}}{d t} \\
& \frac{d \lambda_{d}}{d t}=L_{d}^{\prime} \frac{d i_{d}}{d t}+M_{d}^{\prime} \frac{d i_{q}}{d t}
\end{aligned}
$$

By the equation (2),(3) and equation (4),(5),the electrical models of motor is given by :

$$
\begin{gathered}
v_{d}=r_{s} i_{d}+\frac{d \lambda_{d}}{d t}-\omega_{e}\left(\lambda_{q}-\lambda_{p m}\right) \\
v_{q}=r_{s} i_{q}+\frac{d\left(\lambda_{q}-\lambda_{p m}\right)}{d t}+\omega_{e} \lambda_{d}
\end{gathered}
$$

Since flux linkage caused by the permanent magnet is a constant, equation (7) is further given by

$$
v_{q}=r_{s} i_{q}+\frac{d \lambda_{q}}{d t}+\omega_{e} \lambda_{d}
$$




\section{Analysis of FEA}

The flux linkages can be obtained accurately using the FEA program. This is a software based solution where the physical dimensions and other information about the PMA RSM are entered into the FEA program. The program creates a mesh to partition the object into finite elements. For each element, the vector potential is determined and from all the vector potentials other attributes, the flux linkages calculated are shown in Fig.4.
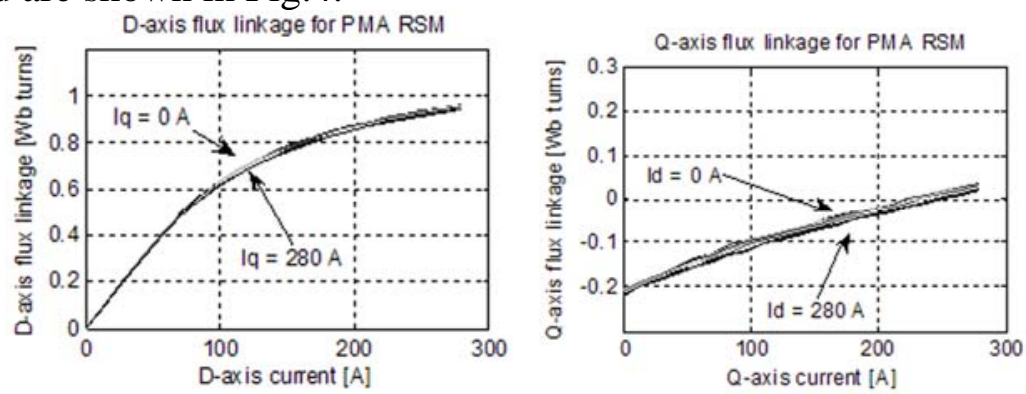

Fig.4 FEA results for D-Q axis flux linkages

From the flux linkages it is possible to calculate self inductances and mutual inductances, the result of which are shown in Fig.5 and Fig.6.
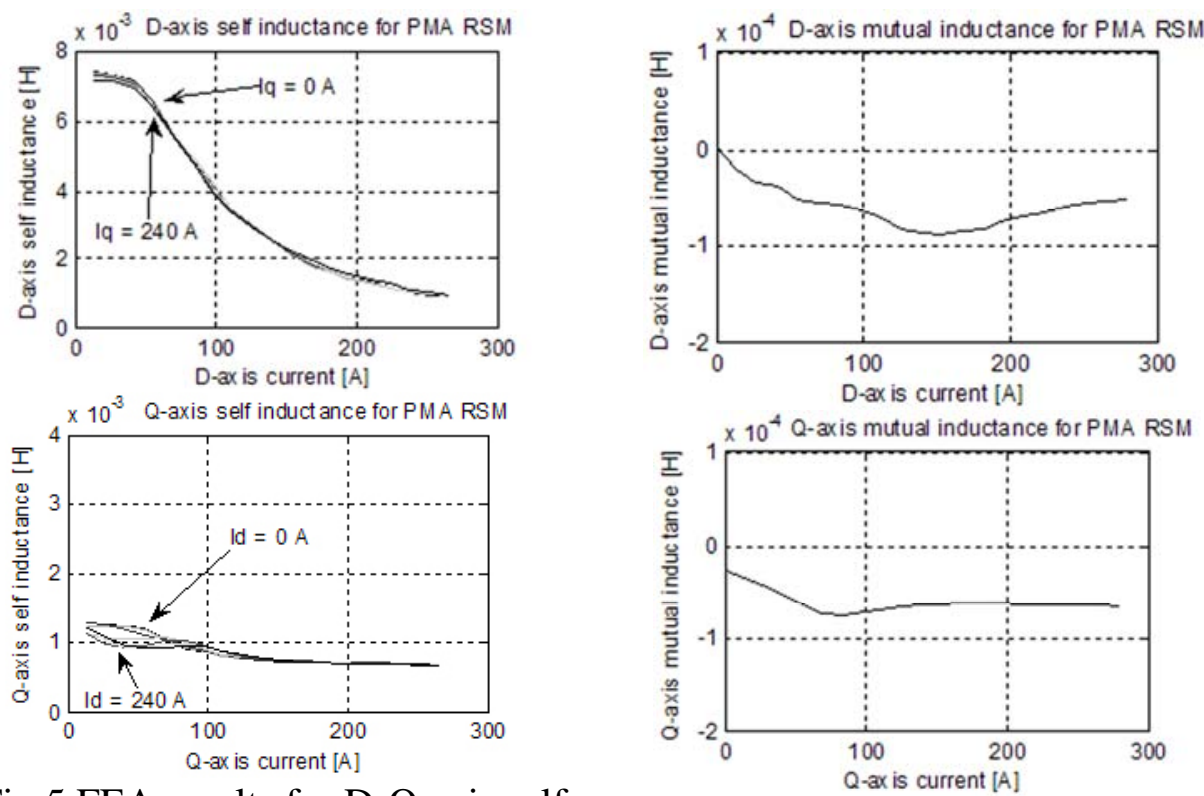

Fig.5 FEA results for D-Q axis self inductances

Fig.6 FEA results for D-Q axis mutual inductances

\section{The simulation of torque response}

Under the control of the constant field current, we take a simulation of the reluctance synchronous motor, The results show that using the torque state observer can obviously improve response of the permanent magnet assisted reluctance synchronous machine The control system of the motor composing one speed loop (including a current loop) is shown in Fig.7.

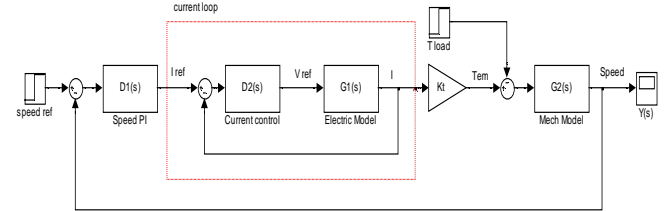

Fig.7 Simulation diagram of the control system

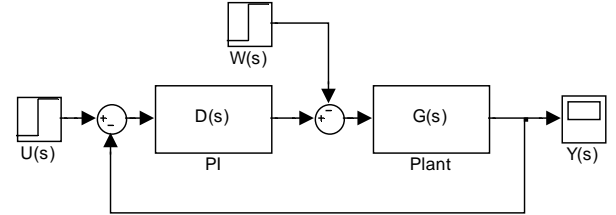

Fig .8 control system diagram 
By the block diagram of figure 8 and the principle of superposition, output equation of the state is given by:

$$
Y(s)=U(s)\left[\frac{D(s) G(s)}{1+D(s) G(s)}\right]-W(s)\left[\frac{G(s)}{1+D(s) G(S)}\right]
$$

By equation (9) shows that the first and second parts of the right equation have the same pole, so have the same response time. Take $D(s)=K_{P}+\frac{K_{I}}{s}=\frac{K_{P} s+K_{I}}{s}, G(s)=\frac{10}{s+10}$ Output equation of the state can be further given by:

$$
Y(s)=U(s) \frac{10\left(K_{P} s+K_{I}\right)}{s(s+10)+10\left(K_{P} s+K_{I}\right)}-W(s) \frac{10 s}{s(s+10)+10\left(K_{P} s+K_{I}\right)}
$$

In equation(10), take $\mathrm{KP}=1, \mathrm{KI}=10$, as $\mathrm{t}=1 \mathrm{~s}$, take $U(s)=\frac{8}{s}$, as $\mathrm{t}=3 \mathrm{~s}$, take $W(\mathrm{~s})=\frac{8}{\mathrm{~s}}$, The simulation of torque response is shown in Fig.9.It can be seen the torque response of a permanent magnet assisted reluctance synchronous machine is faster from the simulation results shown in Fig.9.

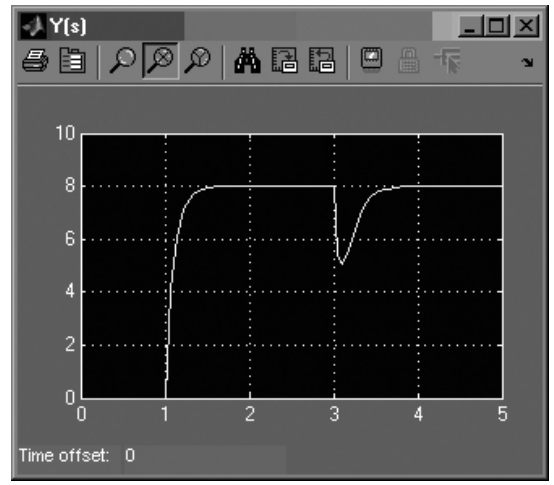

Fig.9 Electrical torque response of the Permanent Magnet Assisted reluctance synchronous machine

\section{Conclusions}

Permanent magnet embedded in the rotor slot plate weakened to the magnetic effect. the D-axis self inductance is greater than the Q-axis self inductance and therefore the D-axis current response is slower than the Q-axis current response. With constant field control, it is only the Q-axis current that needs to change and therefore this method gives a fast current and torque response.

\section{References}

[1] Sibande, S.E., "Design and Perfomance Evaluation of a PM-Assisted Rotor of a 110kW Reluctance Synchronous Machine”, M.Sc.Eng Dissertation, University of Stellenbosch, 2004.

[2] Franklin G., Powell J. and Emami-Naeini A. "Feedback control of Dynamic Systems". Prentice Hall, 2002.

[3] Kamper M.J. and Mackay A.T.:“Optimum Control of the Reluctance Synchronous Machine with Cageless Flux Barrier Rotor”, SAIEE Trans., June 1995, Vol. 86, No. 2, pp. 49-56.

[4] Fratta A. and Vagati F.:"Synchronous Reluctance vs Induction Motor: a comparison”, Proceedings Intelligent Motion (Nürnberg), April 1992, pp. 179-186.

[5] Bombela X.B., Jackson S.K. and Kamper M.J.: "Performance of Small and Medium Power Flux Barrier Rotor Reluctance Synchronous Machine Drives”, ICEM (Istanbul), Sept. 1998, Vol. I, pp. 95-99. 